\title{
Discourse in Mild Cognitive Impairment
}

\author{
Malin Antonsson ${ }^{1}$, Kristina Lundholm Fors ${ }^{1}$, Dimitrios Kokkinakis ${ }^{1,2}$ \\ ${ }^{1}$ The Swedish Language Bank, Dept. of Swedish, Uni. of Gothenburg, Sweden \\ ${ }^{2}$ Centre for Ageing and Health - AgeCap, University of Gothenburg, Sweden \\ https://doi.org/10.36505/ExLing-2019/10/0005/000367
}

\begin{abstract}
This paper reports on how persons with mild cognitive impairment (MCI) perform on two types of narrative tasks compared to a group of healthy controls (HC). The first task is a widely used picture description task and the other task is a more complex discourse task. Since the latter task puts higher demands on cognitivelinguistic skills, as seen in previous research, we expected this task to be more efficient in discriminating between the two groups. The results confirm this hypothesis.
\end{abstract}

Key words: mild cognitive impairment, dementia, narrative language

\section{Introduction}

Dementia is a neurodegenerative disorder that is increasing in prevalence as the global population ages. Mild cognitive impairment $(\mathrm{MCI})$ is a condition that is regarded as a stage preceding dementia (Reisberg \& Gauthier 2008). Linguistic analysis has shown great potential in identifying early signs of dementia, since the disorder negatively affects language production at all levels (Lancashire \& Hirst 2009). Although linguistic features are more successful in identifying persons with clinically manifest dementia than persons with MCI (see e.g. López-de Ipiña et al. 2013), a deterioration in both vocabulary and syntax can be seen several years before a dementia diagnosis (Le et al. 2011). However, differences between healthy controls and persons with MCI are subtle and sometimes difficult to capture (Lundholm Fors et al. 2018; Fraser et al. 2018).

Research has shown that persons with MCI need complex tasks in order for their linguistic difficulties to become evident, as they are able to compensate for their linguistic deficits to some extent (Mueller et al. 2018). Fleming (2014) used a so-called 'planning task' to elicit complex discourse. Although this task is suggested to be more sensitive to language decline than a picture elicited narrative, no study has yet to our knowledge compared the two types of tasks for this population. Hence, the purpose of this paper is to present and compare some preliminary results of the linguistic analyses on the two tasks.

\section{Method}

\section{Participants}

A total of 23 patients with MCI and a group of healthy controls $(\mathrm{HC} ; \mathrm{n}=34)$ participated in the study. A significant difference between the groups was seen

ExLing 2019: Proceedings of 10 $0^{\text {th }}$ International Conference of Experimental Linguistics, 25-27 September 2019, Lisbon, Portugal 
in the Mini Mental State Examination (a test of cognitive functioning), but not on age (mean age in years: MCI: 71.7, HC: 69.2) or education (mean education in years: MCI 13.9, HC 13.3). Detailed inclusion and exclusion criteria are described in Kokkinakis et al. (2017). The study was approved by the local ethical committee review board (reference number: 206-16, 2016).

\section{Data and statistical analyses}

The tasks consisted of a picture description task and a narrative discourse task. The aim of the picture description task was to describe the Cookie theft picture (from BDAE; Goodglass \& Kaplan, 1983). The discourse task is modelled on the 'Trip to New York' task described in Fleming (2014). In brief, the participants were asked to describe how they would prepare for and execute a trip to Stockholm. The audio recordings were manually transcribed and automatically annotated using Sparv (Borin et al. 2016). Alignment of the audio recordings and transcriptions was made using Webmaus (Kisler et al. 2017), with post-corrections done manually.

Since a recent review (Filiou 2019) suggested outcome measures should include aspects of speech production, fluency and semantics, both tasks were analysed and compared on production length (number of words and number of sentences), pronoun use (pronoun index i.e. pronoun / pronoun + nouns), and some aspects of fluency (ratio of false start to number of words and ratio of fillers to number of words). To investigate differences between participants with MCI and $\mathrm{HC}$ we compared the groups using a MANOVA with the abovementioned variables as dependent variables.

\section{Results}

In the cookie theft task, the groups showed no significant differences $(\mathrm{F}(5$, $50)=0.47, p=0.80)$, whereas the groups differed on their performance on the planning task $(\mathrm{F}(5,50)=2.68, \mathrm{p}=0.03)$; see details in table 1 . The groups predominantly differed on aspects of productivity, where the MCI group produced fewer words and a smaller number of sentences than the HC group. Although the MCI group had a higher pronoun index and a lower false start ratio than the HC group in the planning task, these differences were not statistically significant. The number of fillers relative to the number of words did not differ between the groups.

Table 1. Comparison of the two groups on Cookie theft and Planning task.

\begin{tabular}{|l|l|l|l|l|l|l|}
\hline \hline & \multicolumn{3}{|l|}{ Cookie theft } & \multicolumn{3}{l|}{ Planning task } \\
\hline & MCI & HC & sig. & MCI & HC & sig. \\
\hline Words (n) & 142.0 & 172.7 & n.s & 325.4 & 426.4 & $\mathrm{t}=5.8, \mathrm{p}=0.019$ \\
\hline Sentences (n) & 11.5 & 12.7 & n.s & 22.8 & 30.7 & $\mathrm{t}=8.18, \mathrm{p}=0.006$ \\
\hline Pronoun index & 0.36 & 0.36 & n.s & 0.55 & 0.58 & n.s \\
\hline False start ratio & 0.005 & 0.005 & n.s & 0.006 & 0.01 & n.s. \\
\hline Fillers ratio & 0.02 & 0.02 & n.s & 0.03 & 0.03 & n.s. \\
\hline \hline
\end{tabular}




\section{Discussion}

Whereas the chosen linguistic features did not reveal any differences when comparing the performance of persons with MCI and $\mathrm{HC}$ on the cookie theft task, the analysis of the planning task reveals a significant difference between the two groups. This supports the hypothesis that the latter puts higher demands on cognitive-linguistic skills and therefore is better in discriminating between persons with MCI and healthy controls. The persons with MCI produced fewer words and fewer sentences than the HC group, a result similar to Fleming \& Harris (2008), that related the shorter length in the MCI group to decreased competence in planning, problem-solving, and organization. Somewhat puzzling this difference was not seen in their more recent study (Fleming 2014) where no difference in length was observed, but in semantic content. The measure used for semantic content in this study, the pronoun index, did not indicate any differences between the groups.

In future studies we will evaluate a larger number of features describing semantic performance, to explore whether differences can be captured with a fine-grained analysis. Furthermore, since the MCI group produced shorter narratives, we plan to analyse the semantic content through thematic coding (i.e. identification of core elements based on Fleming et al. 2008) and frame analysis using Swedish FrameNet++ (Borin et al. 2010).

\section{Acknowledgements}

This work has received support from The Swedish Foundation for Humanities and Social Sciences (grant agreement no: NHS 14-1761:1).

\section{References}

Borin, L., Dannélls, D., Forsberg, M., Toporowska Gronostaj, M., Kokkinakis, D. 2010. Swedish FrameNet++. Proceedings of 3rd SLTC- Linköping, Sweden.

Borin, L., Forsberg, M., Hammarstedt, M., Rosen, D., Schumacher, A., Schäfer, R. 2016. Sparv: Språkbanken's corpus annotation pipeline infrastructure. Proceedings of 6th SLTC. Umeå, Sweden.

Filiou, R-P., Bier, N., Slegers, A. Houzé, B., Belchior, P., Brambati, S. M. 2019. Connected speech assessment in the early detection of Alzheimer's disease and mild cognitive impairment: a scoping review. Aphasiology.1-33.

Fleming, V.B., Harris, J.L. 2008. Complex discourse production in mild cognitive impairment: Detecting subtle changes. Aphasiology. 22:7-8, 729-740.

Fleming, V.B. 2014. Early detection of cognitive-linguistic change associated with mild cognitive impairment. Comm. Disorders Quarterly. 35(3), 146-157.

Fraser, K.C., Lundholm Fors, K., Eckerström, M., Themistocleous, C., Kokkinakis, D. 2018. Improving the sensitivity and specificity of MCI screening with linguistic information. In RaPID-2@LREC. Japan.

Goodglass, H., Kaplan, E., Barresi, B. 1983. Boston Diagnostic Aphasia Examination. Philadelphia: Lippincott Williams \& Willkins. W. Kluwer. 


\section{M. Antonsson, K. Lundholm Fors, D. Kokkinakis}

Kisler, T., Reichel U.D., Schiel, F. 2017. Multilingual processing of speech via web services. Computer Speech \& Language. Vol. 45: 326-347.

Kokkinakis D., Lundholm Fors, K., Björkner, E., Nordlund, A. 2017. Data collection from persons with mild forms of cognitive impairment and healthy controls infrastructure for classification and prediction of dementia. NoDaLiDa. 131. LiU Electronic Press;172-82.

Lancashire, I., Hirst, G. 2009. Vocabulary Changes in Agatha Christie's Mysteries as an Indication of Dementia: A Case Study. The 19th Rotman Research Institute Conf., Cognitive Aging:Research and Practice. Toronto.

Le, X., Lancashire, I., Hirst, G., Jokel, R. 2011. Longitudinal Detection of Dementia through Lexical and Syntactic Changes in Writing: A Case Study of Three British Novelists. J of Lit and Ling Computing. 26(4): 435-461.

López-de Ipiña, K., Alonso, J-B., Travieso, C.M., Solé-Casals, J., Egiraun, H., FaundezZanuy, M., Ezeiza, A., Barroso, N., Ecay-Torres, M., Martinez-Lage, P., Martinez de Lizardui, U. 2013. On the selection of non-invasive methods based on speech analysis oriented to automatic Alzheimer disease diagnosis. Sensors. 13(5):6730 6745 .

Lundholm Fors, K., Fraser K.C., Kokkinakis D. 2018. Automated syntactic analysis of language abilities in persons with mild and subjective cognitive impairment. Studies in health technology and informatics. 247:705-709.

Mueller, K.D., Koscik, R.L., Hermann, B.P., Johnson, S.C., Turkstra, L.S. 2018. Declines in connected language are associated with very early mild cognitive impairment. Frontiers in ageing neuroscience. 9:437.

Reisberg, B., Gauthier, S. 2008. Current evidence for subjective cognitive impairment (SCI) as the pre-mild cognitive impairment (MCI) stage of subsequently manifest Alzheimer's disease. Int Psychogeriatr. 20:1-16.

Szatloczki, G., Hoffmann, I., Vincze, V., Kalman, J., Pakaski, M. 2015. Speaking in Alzheimer's Disease, is that an early sign? Importance of changes in language abilities in Alzheimer's disease. Frontiers in Aging Neuroscience. 7, 195.

Wendelstein, B., Stegmeier, J., Frankenberg, C., Felder, E., Schröder, J. 2015. Changes in the use of pronouns in spoken language in the course of preclinical to early stages of Alzheimer's disease. Alzheimer's \& Dementia: J of the Alzheimer's Association. 11(7):784-P5. 